\title{
High applicability of ASO-RQPCR for detection of minimal residual disease in multiple myeloma by entirely patient-specific primers/probes
}

\author{
Yinlei Bai, Kwan Yeung Wong, Tsz Kin Fung and Chor Sang Chim *
}

\begin{abstract}
Allele-specific oligonucleotide real-time quantitative PCR (ASO-RQPCR) is a standardized technique for detection and monitoring of minimal residual disease (MRD) in acute lymphoblastic leukemia (ALL) but not multiple myeloma (MM) due to a low applicability inherent with presence of somatic hypermutation. Herein, by a staged PCR approach and sequencing, clonality and tumor-specific complementarity-determining region 3 (CDR3) sequence were identified in 13/13 MM by sequential PCR of IgH VDJ $(n=10)$, IgH DJ $(n=2)$, or IgK VJ $(n=1)$. Using consensus primers/probes conventionally employed in ALL, ASO-RQPCR worked in three (23.1\%) cases only. Conversely, using entirely patient-specific primers/probes, ASO-RQPCR was applicable in eight (61.5\%) cases with a sensitivity of $5 \times 10^{-4}-10^{-5}$. Moreover, using standard curves constructed by serial dilution of plasmids cloned with patient-specific CDR3, ASO-RQPCR was successful in $12\left(92.3 \%\right.$ ) cases with a sensitivity of $10^{-4}-10^{-5}$, but not in a case lacking an N region, in which design of a tumor-specific ASO primer was precluded. Finally, in a patient in complete response (CR), further reduction of MRD after autologous stem cell transplantation (ASCT) was demonstrated. In summary, using entirely patient-specific primers/probes, ASO-RQPCR was applicable in $>90 \% \mathrm{MM}$ patients and enabled detection of dynamic changes of MRD before and after ASCT despite conventional CR.
\end{abstract}

Keywords: Multiple myeloma, Minimal residual disease, Patient-specific ASO-RQPCR

\section{Letter to the editor}

Minimal residual disease (MRD) in multiple myeloma (MM) may be studied by allele-specific oligonucleotide real-time quantitative PCR (ASO-RQPCR), first applied in acute lymphoblastic leukemia (ALL) using patient-specific ASO forward primers derived from complementarity-determining region 3 (CDR3) sequences of immunoglobulin (Ig) genes [1], combined with consensus reverse germline primers and TaqMan probes in the $\mathrm{J}_{\mathrm{H}}$ region [2]. While applicable in $>90 \%$ ALL [3], the consensus primers/probes are only applicable in 42-75\% MM due to somatic hypermutations $[4,5]$.

Herein, in 13 consecutive MM (Additional file 1: Table S1), clonality and sequence of CDR3 were identified in diagnostic BM of all 13 (100 \%) cases by a staged

\footnotetext{
* Correspondence: jcschim@hku.hk

Department of Medicine, Queen Mary Hospital, The University of Hong Kong, Pokfulam, Hong Kong
}

PCR approach, involving sequential PCR of IgH VDJ, (53.8 \%) by FR1 IgH VDJ PCR, two (15.4\%) by IgH DJ PCR, and one $(7.7 \%)$ by IgK VJ PCR. Similar to previous MM studies [5-7], $\mathrm{V}_{\mathrm{H}} 1, \mathrm{~V}_{\mathrm{H}} 3$, and $\mathrm{V}_{\mathrm{H}} 4$ families were mostly used (12 19\%, 47 63\%, and 16 20\%, respectively) (Additional file 2: Table S3).

ASO-RQPCR was first performed using ASO forward primers and consensus primers/probes, according to the EuroMRD guidelines (Additional file 2: Figure S2; Table S2) $[2,8,9]$. By standard curves serially diluted from diagnostic BM, ASO-RQPCR was applicable in only three $(23.1 \%)$ cases, with a sensitivity of $5 \times 10^{-4}-10^{-4}$ and a quantitative range $(\mathrm{QR})$ of $10^{-3}-10^{-4}$ (Table 1), suggestive of MRD quantification in follow-up samples with one myeloma cell 
Table 1 Performance of RQPCR by consensus germline or entirely patient-specific primers/probes

\begin{tabular}{|c|c|c|c|c|c|c|c|c|c|c|c|c|}
\hline \multirow[t]{3}{*}{ Cases } & \multirow{2}{*}{\multicolumn{4}{|c|}{$\begin{array}{l}\text { Consensus germline primers/probes } \\
\text { Diagnostic marrow DNA as standards }\end{array}$}} & \multicolumn{8}{|c|}{ Patient-specific primers/probes } \\
\hline & & & & & \multicolumn{4}{|c|}{ Diagnostic marrow DNA as standards } & \multicolumn{4}{|c|}{ CDR3-cloned plasmid as standards } \\
\hline & Sensitivity & QR & $R^{2}$ & Slope & Sensitivity & QR & $R^{2}$ & Slope & Sensitivity & QR & $R^{2}$ & Slope \\
\hline $\mathrm{D} \times 53$ & N/A & $10^{-2}$ & $\mathrm{~N} / \mathrm{A}$ & $\mathrm{N} / \mathrm{A}$ & $10^{-5}$ & $10^{-4}$ & 0.996 & -3.30 & $10^{-5}$ & $10^{-4}$ & 0.992 & -3.44 \\
\hline M3 & ND & ND & ND & ND & $5 \times 10^{-4}$ & $5 \times 10^{-4}$ & 0.989 & -3.48 & $10^{-4}$ & $10^{-4}$ & 0.997 & -3.54 \\
\hline M8 & $10^{-4}$ & $5 \times 10^{-4}$ & 0.985 & -3.38 & $10^{-4}$ & $10^{-3}$ & 0.999 & -3.46 & $10^{-4}$ & $5 \times 10^{-4}$ & 0.999 & -3.61 \\
\hline M4 & ND & ND & ND & ND & $10^{-4}$ & $10^{-3}$ & 0.984 & -3.60 & $10^{-5}$ & $10^{-3}$ & 0.999 & -3.54 \\
\hline Dx31 & ND & ND & ND & ND & $10^{-4}$ & $5 \times 10^{-4}$ & 0.998 & -3.45 & $10^{-4}$ & $10^{-4}$ & 0.998 & -3.47 \\
\hline M5 & $10^{-4}$ & $10^{-4}$ & 0.995 & -3.41 & $5 \times 10^{-4}$ & $5 \times 10^{-4}$ & 0.995 & -3.67 & $10^{-5}$ & $10^{-4}$ & 0.998 & -3.51 \\
\hline M10 & N/A & $5 \times 10^{-4}$ & N/A & N/A & N/A & $5 \times 10^{-4}$ & N/A & N/A & $10^{-4}$ & $10^{-4}$ & 0.994 & -3.51 \\
\hline Dx49 & $5 \times 10^{-4}$ & $10^{-3}$ & 0.982 & -3.37 & $10^{-4}$ & $10^{-3}$ & 0.986 & -3.26 & $10^{-4}$ & $5 \times 10^{-4}$ & 0.999 & -3.76 \\
\hline M1 & ND & $\mathrm{ND}$ & ND & ND & $5 \times 10^{-4}$ & $10^{-3}$ & 0.986 & -3.48 & $10^{-4}$ & $5 \times 10^{-4}$ & 0.998 & -3.52 \\
\hline M6 & ND & ND & ND & ND & N/A & $5 \times 10^{-4}$ & N/A & N/A & $10^{-5}$ & $5 \times 10^{-4}$ & 0.997 & -3.72 \\
\hline M2 & N/A & N/A & N/A & N/A & ND & ND & ND & ND & ND & ND & ND & ND \\
\hline M7 & N/A & $10^{-2}$ & N/A & N/A & N/A & $10^{-2}$ & N/A & N/A & $10^{-4}$ & $10^{-3}$ & 0.999 & -3.56 \\
\hline M9 & $\mathrm{N} / \mathrm{A}$ & $10^{-2}$ & $\mathrm{~N} / \mathrm{A}$ & N/A & N/A & $10^{-2}$ & N/A & $\mathrm{N} / \mathrm{A}$ & $10^{-4}$ & $10^{-4}$ & 0.997 & -3.55 \\
\hline
\end{tabular}

Abbreviations: $Q R$ quantitative range, $R^{2}$ correlation coefficient, $N / A$ not applicable, $N D$ not done due to absence of target band in qualitative PCR or no need (for $\mathrm{M} 2$ in patient-specific approach)

in a background of $10^{4}$ normal cells [10]. Causes of failures in other cases included absence of amplification $(n=5)$ or non-specific amplification in normal control $(n=1)$ during preliminary qualitative PCR, suboptimal QR of $10^{-2}(n=3)$, or shortage of diagnostic BM for standard curve $(n=1)$.

To account for a low applicability using consensus primers/probes, a systematic search for sequences mismatches against consensus primers/probes was performed. Sequencing data revealed mismatches in five (50 \%) cases against consensus primers, six (50\%) against probes, and two (20 \%) against both sites, but not in two IgH cases (M7 and M10) or one IgK case
(M9) (Table 2). While a previous MM study also showed a high frequency of mismatches against consensus primers/probes in patients with IgH VDJ rearrangements (herein 80 vs. $75 \%$ ), the number of mismatches per case was higher in our study (five vs. three) [11]. Given that mismatches of $\geq 3$ bases would preclude a successful ASO-RQPCR [11], the use of patient-specific primers/probes is inevitable in Chinese MM with profound mismatches than Caucasian patients.

Therefore, ASO-RQPCR using entirely patient-specific primers/probes was performed (Additional file 2: Figure S2; Table S4). Except one case (M2) amplified by IgH DJ PCR,

Table 2 Mismatches against consensus germline reverse primers and/or probes

\begin{tabular}{|c|c|c|c|c|c|c|}
\hline \multirow[t]{2}{*}{ Cases } & \multicolumn{3}{|c|}{ Mismatches against primers } & \multicolumn{3}{|c|}{ Mismatches against probes } \\
\hline & Primers & Primer binding site sequences & $\overline{\mathrm{No}}$. & Probes & Probe binding site sequences & $\overline{\text { No. }}$ \\
\hline$D \times 53$ & R-JH6-intron & gcagaGaGGaaGggccctagagt & 4 & T-JH6 & cacggtcaccgtctcctcaggCaagaa & 1 \\
\hline M3 & $\mathrm{R}-\mathrm{JH} 4$-intron & cagagAAaaTATCACagagaggttgt & 8 & Т-JH1.2.4.5 & ccctggtcaccgtctcctcaggtg & 0 \\
\hline M8 & $\mathrm{R}-\mathrm{JH} 4$-intron & cagagCtaaagcaggagagaggttgt & 1 & Т-JH1.2.4.5 & ccctggtcaccgtctcctcaggtg & 0 \\
\hline M4 & $\mathrm{R}$-JH4-intron & cagGAttTaagTaggGgagaCgttgt & 6 & Т-JH1.2.4.5 & ccctggtcaccgtctcctcaggtg & 0 \\
\hline Dx31 & $\mathrm{R}-\mathrm{JH} 4$-intron & TagaCCCaaagTaggagagaCAttAt & 8 & Т-JH1.2.4.5 & Tcctggtcaccgtctcctcaggtg & 1 \\
\hline M5 & $\mathrm{R}$-JH3-intron & aggcagaaggaaagccatcttac & 0 & Т-JH3 & cCagggacaatggtcaccgtctcttca & 1 \\
\hline M10 & R-JH6-intron & gcagaaaacaaaggccctagagt & 0 & Т-JH6 & cacggtcaccgtctcctcaggtaagaa & 0 \\
\hline Dx49 & R-JH6-intron & - & $?$ & Т-JH6 & cacggtcaccgtctcctcaggtaagaT & 1 \\
\hline M1 & $\mathrm{R}$-JH4-intron & - & $?$ & Т-JH1.2.4.5 & cTGt- & $\geq 2$ \\
\hline M6 & $\mathrm{R}-\mathrm{JH} 3$-intron & - & $?$ & Т-JH3 & caagggacaat- & $?$ \\
\hline M2 & $\mathrm{R}-\mathrm{JH} 4$-intron & cagagttaaagcaggagagaggttgt & 0 & Т-JH1.2.4.5 & ccctggtcGccgtctcctcaggtg & 1 \\
\hline M7 & $\mathrm{R}$-JH5-intron & agagagggggtggtgaggact & 0 & T-JH1.2.4.5 & ccctggtcaccgtctcctcaggtg & 0 \\
\hline M9 & R-Jk1 & gatcacttcatagacacagggaacag & 0 & T-Jk1 & tggaaatcaaacgtgag & 0 \\
\hline
\end{tabular}

Uppercase letters in sequences indicate mismatches. "?" indicates mismatches cannot be identified based on available sequencing data 
where the absence of an $\mathrm{N}$ region precluded design of a specific ASO primer, patient-specific primers/probes ASORQPCR was successful in eight $(61.5 \%)$ cases (Table 1$)$.

Moreover, as diagnostic BM was limited, we attempted to construct standard curves by plasmid DNA cloned with patient-specific CDR3, whereby ASO-RQPCR was applicable in all 12 cases, with a sensitivity of $\leq 10^{-4}$ in all $12(100 \%)$ cases and a QR $\leq 5 \times 10^{-4}$ in ten $(83.3 \%)$ cases (Table 1). Collectively, using CDR3-cloned plasmid DNA, more cases achieved a sensitivity of $\leq 10^{-4}$ than using diagnostic BM (12 vs. 5 cases: $p=0.0046$; Table 1 ), and applicability was increased from 61.5 to $92.3 \%$. Moreover, plasmid DNA provides an unlimited supply for standard curve construction in multiple MRD analysis. Furthermore, the use of plasmid DNA is more accurate for cases in which the percentage of plasma cells was not specified in the diagnostic BM.

Finally, in a patient (Dx53) who achieved serological complete response (CR) after four cycles of VTD induction, by ASO-RQPCR with a sensitivity of $10^{-5}$ and QR of $10^{-4}$, MRD was present in both BM samples on days 120 and 16 prior to autologous stem cell transplantation (ASCT), but turned negative on day 14 after ASCT, testifying further reduction of tumor load below clinical detection limit by ASCT (Additional file 2: Figure S3).

In summary, a high applicability and sensitivity of MRD detection by ASO-RQPCR can be achieved by clonality detection using stepwise PCR approach, mismatch analysis, and hence entirely patient-specific primers/probes in addition to standard curve construction using CDR3cloned plasmid DNA. Moreover, the use of ASCT in a patient achieving serological $\mathrm{CR}$ after induction was justified by further reduction of tumor load illustrated by ASO-RQPCR. Finally, the frequency and intensity of mismatches against consensus primers/probes between different populations warrants further study in an expanded cohort.

\section{Additional files}

Additional file 1: Materials and methods and Table S1 patient demographics. (DOCX $67 \mathrm{~kb}$ )

Additional file 2: Figure S1. Schematic diagram. Figure S2. Principles of ASO-RQPCR. Figure S3. MRD detection. Table S2. Clonality and ASO primers. Table S3. Family usage. Table S4. Patient-specific primers/probes and downstream primers. (DOCX $2290 \mathrm{~kb}$ )

\footnotetext{
Abbreviations

ALL: Acute lymphoblastic leukemia; ASCT: Autologous stem cell transplantation; ASO-RQPCR: Allele-specific oligonucleotide real-time quantitative PCR; CDR: Complementarity-determining region; CR: Complete response; Ig: Immunoglobulin; MM: Multiple myeloma; MRD: Minimal residual disease
}

\section{Funding}

Nil.

\section{Availability of data and materials}

Detailed materials and methods and all data are included in this published article and its additional files.

\section{Authors' contributions}

CSC designed the research study. CSC helped in the sample collection and clinical data retrieval. YB and TKF performed the research. CSC, KYW, and YB wrote the paper. All authors analyzed the data and had final approval of the submitted and final versions.

\section{Competing interests}

The authors declare that they have no competing interests.

\section{Consent for publication \\ Not applicable.}

\section{Ethics approval and consent to participate}

The study was approved by the Institutional Review Board of the University of Hong Kong/Hospital Authority Hong Kong West Cluster (HKU/HA HKW IRB, IRB Reference Number: UW 05-269 T/932) with informed consents.

Received: 3 September 2016 Accepted: 4 October 2016

Published online: 11 October 2016

\section{References}

1. Chim JC, Coyle LA, Yaxley JC, Cole-Sinclair MF, Cannell PK, Hoffbrand VA, et al. The use of IgH fingerprinting and ASO-dependent PCR for the investigation of residual disease (MRD) in ALL. Br J Haematol. 1996;92:104-15.

2. Verhagen OJ, Willemse MJ, Breunis WB, Wijkhuijs AJ, Jacobs DC, Joosten SA, et al. Application of germline IGH probes in real-time quantitative PCR for the detection of minimal residual disease in acute lymphoblastic leukemia. Leukemia. 2000;14:1426-35.

3. Bruggemann M, Schrauder A, Raff T, Pfeifer H, Dworzak M, Ottmann OG, et al. Standardized MRD quantification in European ALL trials: proceedings of the Second International Symposium on MRD assessment in Kiel, Germany, 18-20 September 2008. Leukemia. 2010;24:521-35.

4. Sarasquete M, Garcia-Sanz R, Gonzalez D, Martinez J, Mateo G, Martinez P, et al. Minimal residual disease monitoring in multiple myeloma: a comparison between allelic-specific oligonucleotide real-time quantitative polymerase chain reaction and flow cytometry. Haematologica. 2005;90:1365-72.

5. Puig N, Sarasquete ME, Balanzategui A, Martinez J, Paiva B, Garcia H, et al. Critical evaluation of ASO RQ-PCR for minimal residual disease evaluation in multiple myeloma. A comparative analysis with flow cytometry. Leukemia. 2014;28:391-7.

6. Gonzalez D, van der Burg M, Garcia-Sanz R, Fenton JA, Langerak AW, Gonzalez $\mathrm{M}$, et al. Immunoglobulin gene rearrangements and the pathogenesis of multiple myeloma. Blood. 2007:110:3112-21.

7. Owen RG, Johnson RJ, Rawstron AC, Evans PA, Jack A, Smith GM, et al. Assessment of IgH PCR strategies in multiple myeloma. J Clin Pathol. 1996; 49:672-5.

8. van der Velden VH, Cazzaniga G, Schrauder A, Hancock J, Bader P, PanzerGrumayer ER, et al. Analysis of minimal residual disease by $\mathrm{lg} / \mathrm{TCR}$ gene rearrangements: guidelines for interpretation of real-time quantitative PCR data. Leukemia. 2007:21:604-11.

9. van der Velden VH, de Bie M, van Wering ER, van Dongen JJ. Immunoglobulin light chain gene rearrangements in precursor-B-acute lymphoblastic leukemia: characteristics and applicability for the detection of minimal residual disease. Haematologica. 2006:91:679-82.

10. Paiva B, van Dongen JJ, Orfao A. New criteria for response assessment: role of minimal residual disease in multiple myeloma. Blood. 2015;125:3059-68.

11. Gonzalez D, Gonzalez M, Alonso ME, Lopez-Perez R, Balanzategui A, Chillon MC et al. Incomplete DJH rearrangements as a novel tumor target for minimal residual disease quantitation in multiple myeloma using real-time PCR. Leukemia. 2003;17:1051-7. 